\title{
Ethical dilemmas in malaria drug and vaccine trials: a bioethical perspective
}

Michele Barry and Malcolm Molyneux

Yale University School of Medicine, New Haven and Liverpool School of Tropical Medicine, Liverpool, respectively

\section{Authors' abstract}

Malaria is a disease of developing countries whose local health services do not have the time, resources or personnel to mount studies of drugs or vaccines without the collaboration and technology of western investigators. This investigative collaboration requires a unique bridging of cultural differences with respect to human investigation. The following debate, sponsored by The Institute of Medicine and The American Society of Tropical Medicine and Hygiene, raises questions concerning the conduct of trans-cultural clinical malaria research. Specific questions are raised about the difficulties of informed consent in different cultural settings and whether there is any role for community involvement. Discussants debate whether drug and vaccine trials not approved in an industrialised country are ever defensible if performed in a third-world setting. Potential conflicting priorities between investigators are discussed and ideas regarding conflict resolution are offered.

MB Our task is to consider some of the ethical problems that surround drug and vaccine trials for malaria. We propose to do this as a dialogue, because we believe the subject lends itself more to debate than to pronouncement. We also would like to frame this debate around the bioethical principles that guide human investigations, which have been well delineated by the Nuremberg Code and the Helsinki Declaration $(1,2)$.

Dr Molyneux, most people now agree that through clinical trials, and in particular controlled trials, we have been able to make important advances in clinical science. Ethical principles that should govern such trials have been elaborated (3). What is special or different about trials involving malaria research?

MM Malaria is mainly a disease of developing countries. Most of the million or more people who die of malaria every year are young children in endemic areas (4). It is therefore in these areas that most clinical trials of drugs, and in due course vaccines, must be carried out. Local health services cannot be expected to

\section{Key words}

Malaria vaccine trials; ethics; developing countries; informed consent; malaria drug trials have the money, time or personnel to mount studies of drugs or vaccines without the collaboration of scientists from industrial nations. Malaria is therefore a disease that requires for its study a bridging between cultures, a sharing of very different kinds of expertise between nations.

\section{Informed consent and autonomy}

MB Yet a number of ethical difficulties must arise in connection with clinical trials in developing nations. The first has to do with the willingness of subjects to take part in trials. The Nuremberg Code requires that individuals should only be subjected to biomedical research if they, or their responsible representative, give consent freely and without coercion, having beenf adequately informed of the nature and purpose of the study (1). Taking the latter point first, Dr Molyneux, can, for example, an African villager involved in one of your studies fully understand your reasons for wanting to enroll her unconscious child in a study in which he may or may not be given an additional anti-malarial drug? Is she able to make a free assessment of the possible risks versus possible benefits, before giving her consent in that setting?

MM 'Informed' consent is a problem anywhere. In a consensus conference in this country, 79 of 92 participants considered that the obtaining of informed consent was problematic because patients or surrogates could not fully understand the implications of a formal clinical trial (5). When we work in a foreign culture we make sure that potential participants, or their surrogates, receive as clear an explanation as possible in their own language, given to them by a member of the trial team who belongs to that culture. The explanation must be made with due understanding of the individual's educational background, and in the light of local concepts of health and disease (6). Individuals may not understand the science of the study, but it is quite possible for the choice to be presented in a way that they can understand. As Ajayi has said, it is false to equate illiteracy with the inability to take decisions (7).

MB Isn't it true in some cultures people have ideas about health and disease that are incompatible with a scientific explanation? For example, the belief that disease is caused by an aggrieved enemy or ancestor, or 
that to remove a blood-sample is to rob a person of essential vitality?

MM People's beliefs about the causes of disease sometimes reduce recruitment to trials or may make them impossible. But in drug trials the very fact that a patient has come for teatment indicates a willingness to try a scientific remedy.

Many people are suspicious of our attempts to explain disease. (In the case of malaria their suspicions are well founded: although we know about the parasite, we ourselves have very little understanding of the pathogenetic mechanisms of the disease.) Nevertheless investigators must remain sensitive to differences in the way they and their patients understand disease; discussion and explanation may be necessary at intervals during a study, not only at the beginning.

MB Whatever the setting, in both industrial and developing communities, participants in a trial ultimately place their trust in the investigator and believe that he or she is acting in their best interest. Yet there clearly have to be safeguards to ensure that enrolment into a study is culturally comprehensible and not coercive.

What about the act of consent itself? Consent as we understand it requires the decision of a free, autonomous individual - a person able to decide for herself without constraint or undue pressure from others. Is it not true that in many cultures, individual autonomy may be a dynamic system involving the family, group or village (8)? How meaningful is the individual's 'informed consent' in such circumstances? MM For many trials, especially those which will take place within the village setting (for example, vaccine trials), decisions about participation are likely to be made at the level of the extended family, village or even tribal authority. In smallpox vaccine trials (9) and trials of insecticide-spraying (10) investigators reported that once the tribal leaders or elders, or the village as a whole, agreed to a study, it was rare for any individual to decline.

MB So, community involvement may be as important as individual consent in some cultural settings, yet obviously should not override or substitute for an individual's refusal to participate. Given the enormous gap in the level of scientific knowledge between the investigators and the people to be studied, is there not great potential for a kind of paternalistic scientific imperialism in malaria trials? What safeguards are there to ensure that scientists are not able to manipulate subjects into participation in studies that might not be in the subjects' best interests?

MM Almost every country now has its own scientific and ethical review board, to which every proposed trial must be submitted. In these committees professionals or lay-persons within the country may question the justification for a study or the benefit that the results are likely to bring to their own people. There may also be local ethical committees in the hospital or district in which a study is to be done. The existence of these local controls on scientific activity ensures that the proposal has been sanctioned by representatives of the people to be studied. The consenting individual is then placing her trust as much in her own countrymen as in the outsider.

MB Yet often local review or ministry review boards can be responding to political pressures, foreign exchange or the interests of an elite. Double safeguards of both host and recipient independent review may mitigate this problem. What about the subtle pressure on individuals or groups to take part in studies; pressures that might flout the Helsinki requirement for freedom from coercion?

I'm thinking, for example, of the extra clinical care that a mother might expect her child to receive if the child is admitted to a malaria-treatment study that provides not only a trial drug but also intensive care by the research team.

MM In many cultures patients see their relationship with a healer in terms of a contract, and it is entirely reasonable that the contract should be mutually beneficial. The fact that there will be benefits in the form of health care for those entering a particular study is an appropriate part of the contract. It would be unreasonable to expect people to take part in a controlled trial, to put up with the additional sampling or interviewing involved, to have a 50 per cent chance 8 of receiving a placebo with no potential benefit whatever, and yet to have no additional compensation for taking part. In a field-study, such as a vaccine trial, requiring the regular clinical evaluation of those who participate, clinical investigators cannot ignore other medical problems that their patients develop, and some general services may have to be offered. In practice it is usually logistically impossible to offer such services to the entire community; they may have to be offered as part of the contract to those who enroll in the study.

\section{Primum non nocere}

MB Yet it is important to recognise that such services may reflect subtle pressure on an individual to enroll in a study. Leaving the difficult subject of informed consent, I'd like to consider the Hippocratic dictum primum non nocere - in relation to clinical trials in malaria. The Helsinki Declaration emphasises that during the investigation of human subjects, the interest of science and society should never take precedence over consideration of the individual's wellbeing (1).

If we consider trials of a malaria vaccine, is it possible that while a campaign may reduce transmission and benefit the community as a whole, the vaccinated individual may, as artificial immunity wanes, become more susceptible to severe malaria thereafter than he was before being vaccinated?

MM In any vaccine trial this possibility will have to be considered carefully. In the preliminary studies it should be possible to follow recipients closely, to detect any increased susceptibility with time and to provide 
effective treatment for malarial illness. The larger question is whether a successful vaccination programme would convert an area which has holoendemic, stable malaria into one which is subject to epidemic, unstable malaria, where many persons, both vaccinated and unvaccinated, may become susceptible to severe disease.

MB Thus, ongoing monitoring of malaria transmission in vaccinated areas will be crucial and will need to be supported after preliminary studies.

With all drug trials in developing nations, there is a danger that drugs which have not yet passed the stringent safety requirements of industrial nations might be used in populations with less developed regulatory mechanisms.

MM This has happened quite often, in general therapeutic practice as well as in research. But it doesn't need to happen. Investigators should have to satisfy not only the scrutiny of host-country scientific and ethical committees, but also the requirements of both the collaborating institution and the donor of financial support.

MB Yet for example, malaria has high morbidity and mortality in pregnant women, a group that is virtually impossible to test in the United States for any new drug. Some of the earlier mefloquine studies which demonstrated the drug's safety during pregnancy were done by a US agency in a developing nation, yet the drug was subsequently not released in the US for use during pregnancy because of potential teratogenicity (11). Should US investigators be performing drug or vaccine studies on a population that would not be allowed to be tested here in the US?

MM You said that malaria during pregnancy has a high morbidity; efficacious and safe drugs are desperately needed. We should remember that US investigators must submit their research protocols for independent ethical review within their home country as well as in the host country, and the ethical standards applied are equally stringent wherever the work is to be done. The early mefloquine studies were subject to this process. The Food and Drug Administration (FDA) in the United States will, however, rarely license a drug for use in pregnancy without extensive human data. These data for malaria can only be collected in developing nations and sufficient data have not yet been gathered. The requirements of the FDA for licensing a drug in the US are not the same as those that justify trials, and as long as ethical committees approve, trials should continue. The clinical problems are great, and we will not solve them by doing nothing.

\section{Justice}

MB Clinical problems may be great yet we must exercise consistent ethical standards in addressing them. I'd like to move on now to the principle of justice which is that neither the benefits nor the burdens of research should be unjustly distributed. In other words, if the burden of preliminary trials of a drug or vaccine is to be borne by communities in malarious areas, the benefits of the resulting knowledge should be available to the same communities. Studies in a developing country may prove that a product is highly effective, but the knowledge may be useless to that country, if it cannot afford to buy supplies of the product. Meanwhile the richer world may have gained useful information for its travellers or soldiers. Would this not be a violation of the principle of justice?

MM Studies should be designed so that the host country can benefit from what is learned. But 0 sometimes the benefit may not be immediate. For example, a successful trial may lead to a product becoming steadily cheaper as it is produced on a large scale, as happened with chloroquine. Some trials actually prove the lack of efficacy of a commonly used treatment, with the result that useless expenditure is saved, as in the studies that showed dexamethasone to be of no value in the treatment of cerebral malaria $(12,13)$.

MB Yet in order to be ethically responsible, 오 collaborative trials in developing nations should address the health priorities of the host country and not divert resources or manpower from more pressing health needs in order to study a question perhaps of more interest to the western collaborator. It seems particularly possible that a malaria vaccine, although tested in the field in populations in endemic areas might then be of greater benefit to the investigato than to the investigated. It may be easy to give a vaccina to travellers from rich nations but economically an logistically difficult to administer it to entire populations in poorer countries.

MM This is a strong possibility. What is more likely is that a new vaccine will be introduced with enthusiasm but it will not be possible to sustain its distribution over time in areas in need of it. If a successful vaccine is developed, developed nations will need to devote considerable assistance, in funds and facilities, to help developing countries to sustain vaccination programmes. It will be unethical if rich nations fail to do this.

MB There is another aspect of justice which we should consider. It concerns not the population at risk of malaria, but the scientists and professional providers of health in countries where malaria is a major endemic $\rightarrow$ disease. They too are at risk of exploitation. Is there any guarantee that when research studies are done by $N$ outside teams, some expertise is passed on to members of the host nation who have to remain and continue to work in their own country?

MM The World Health Organisation's Training in Disease Research (TDR) programme aims to strengthen the capability of host countries to do research. Funds are given to promote collaborative work which includes a training component. All $\frac{7}{0}$ research - and indeed all service-programmes in which $\stackrel{\circ}{\mathbb{D}}$ rich and poor collaborate - should include a strong training function, so that the host country inherits not $\stackrel{\mathbb{D}}{\circ}$ only the results of research but the capacity to identify and effectively study its own problems. It is all too 
common for an outside team to practise 'smash and grab' research, gathering data of interest to themselves or of benefit to their careers, and then disappearing without allowing their hosts the opportunity to participate in or carry forward the work, much less to benefit from it.

MB Clearly there are complexities, both ethical and logistic, in studying malaria. Our expenditure in this field is so small in relation to the enormous size of the disease as a worldwide problem. Perhaps $\mathrm{Dr}$ Molyneux, you want to comment upon what compels us to study a disease which is of so little risk to the developed world.

MM There may be a lot of difficulties in doing ethically justifiable studies in the prevention and treatment of malaria. But I am quite convinced of this: that there are many of these studies that it would be unethical not to do. There is still a shamefully high morbidity and mortality from this disease. The mortality of cerebral malaria in a child in Africa, even with the best current therapy, is high -37 per cent in those with profound coma (14). Thousands of children die every month from malarial anaemia. Parasites have developed at least some resistance to each of our meagre range of therapeutic drugs. We must be sensitive to the needs and cultures of those most affected by malaria but we must contribute all we can to combating this most important of all parasitic infections.

MB Our hope with this exchange was to raise more questions for discussion rather than to issue ethical dictates. We would like to encourage comments from the audience during the panel sessions later. I think we would both agree with Cabot, who said 'Ethics and science must shake hands'. It's time you and I did the same.

The debate was held in New Orleans, Louisiana, USA, during the annual meeting of the American Society of Tropical Medicine, in December 1990.

Michele Barry, MD, FACP, is Associate Professor of Medicine, International Health Program, Yale University School of Medicine, New Haven, USA. Malcolm Molyneux, MB, BChir, MD, FRCP, is Senior Lecturer in Tropical Medicine and Honorary Consultant Physician in the Liverpool School of Tropical Medicine,
Liverpool, England.

\section{References}

(1) Beauchamp T L, Childress J F, eds. Principles of biomedical ethics. (2nd ed.) Oxford: Oxford University Press, 1983; 339-343.

(2) Proposed international guidelines for biomedical research involving human subjects. Geneva: World Health Organisation 1981: 1-49.

(3) CIOMS. International guidelines for ethical review of epidemiological studies. Law, medicine and health care $1991 ; 19,3-4:$ 247-258.

(4) World Health Organisation. World malaria situation, 1983. World health status quarterly 1985; 38: 193-231.

(5) Blum A L, Chalmers T C, Deutsch J et al. The Lugano statement on controlled clinical trials. Fournal of international medical research 1987; 15: 2-22.

(6) Barry M. Ethical considerations of human investigation in developing countries: the AIDS dilemma. New England journal of medicine 1988; 319: 1083-1086.

(7) Ajayi O O. Taboos and clinical research in West Africa. Fournal of medical ethics 1980; 6: 61-63.

(8) De Craemer W. A cross-cultural perspective on personhood. Milbank memorial fund quarterly 1983; 63: 19-34.

(9) Henderson D A et al. Assessment of vaccination coverage, vaccine scar rates and smallpox scarring in five areas of West Africa. Bulletin of the World Health Organisation 1973; 84: 183-194.

(10) Hall A J. Public health trials in West Africa: logistics and ethics. Investigative review board 1989; 11: 8-10.

(11) Steketee R W, Wirima J J, Heymann D C, Khoromana C, Bremen J G. Efficacy of mefloquine and chloroquine prophylaxis in pregnancy. Centers for Disease Control, Atlanta, Georgia; Ministry of Health, Lilongwe, Malawi. Presented at the 38th Annual Meeting of the American Society of Tropical Medicine and Hygiene, 1989 Dec 10-14: Abstract 283: 222.

(12) Warrell D A, Looareesuwan S, Warrell M J et al. Dexamethasone proves deleterious in cerebral malaria: a double blind controlled trial in 100 comatose patients. New England journal of medicine 1982; 306: 313-319.

(13) Hoffman S L, Rustama D, Punjabi N H et al. High dose dexamethasone in quinine-treated patients with cerebral malaria: a double-blind, placebo-controlled trial. Fournal of infectious diseases 1988; 158,2: 325-331.

(14) Molyneux M E, Taylor T E, Wirima J J, Borgstein A. Clinical features and prognostic indicators in paediatric cerebral malaria: a study of 131 comatose Malawian children. Quarterly jourmal of medicine 1989; 71,265: $441-459$. 